\title{
The Role of Atezolizumab in the Treatment of Triple- Negative Breast Cancer
}

\author{
Paulo Luz*, Luis Bretes and Ana Julia Arede \\ Centro Hospitalar Universitário do Algarve, Medical Oncology, CBIOS - Universidade Lusófona's Research Center for Biosciences \& Health Technolo- \\ gies, Lisboa, Portugal
}

${ }^{\star}$ Corresponding author: Paulo Luz, Centro Hospitalar Universitário do Algarve, Medical Oncology, CBIOS - Universidade Lusófona’s Research Center for Biosciences \& Health Technologies, Lisboa, Portugal

Received: March 03, 2021; Accepted: March 08, 2021; Published: March 15, 2021

\begin{abstract}
Triple-Negative Breast Cancer (TNBC) is commonly treated with chemotherapy. However, immunotherapy has been widely suggested as a treatment option for patients with TNBC, including atezolizumab. The present narrative article aims to fully understand the evidence of atezolizumab in the treatment of TNBC. Newer and better biomarkers are needed to select patients with TNBC that are more likely to benefit from immunotherapy.
\end{abstract}

Keywords: Atezolizumab, Immunotherapy, Breast cancer, Tumor-infiltrating lymphocytes

\section{Introduction}

Triple-Negative Breast Cancer (TNBC) consists in a very aggressive breast cancer, often including earlier recurrence and metastasis, that is essentially characterized by the lack of progesterone, estrogen, and human epidermal growth factor receptor-type 2 (HER2), accounting for about $15 \%$ up to $20 \%$ of all the breast cancers. The most common therapy for the metastatic TNBC is chemotherapy, even though it refers to a short-lived type of responses, considering that patients frequently present a median overall survival of 12 up to 18 months. Hence, there is a certain need to develop further studies to improve the existing therapies or to introduce innovative ones [1].

According to previous studies, immunotherapy actually represents a very promising treatment for TNBC mainly due to the fact that TNBC has more tumor-infiltrating lymphocytes (TILS), higher levels of Programmed Death Ligand 1 (PD-L1) expression on immune cells and the tumor, and a greater number of nonsynonymous mutations [2-4]. Atezolizumab consists in an Fcengineered, humanized immunoglobulin G1 monoclonal antibody which is expressed on tumor cells and on tumor-infiltrating immune cells. Basically, this agent directly binds to PD-L1 and blocks its interaction with the Programmed Death Protein 1 (PD-1), while simultaneously enabling the reactivation of the anti-tumor immune response without the antibody-dependent cytotoxicity [5]. The present article reviews some of the main studies that suggest the use of atezolizumab in the treatment of TNBC due to its efficiency and positive outcomes among patients with this disease. The main goal is to fully understand the evidence of the use of atezolizumab in the treatment of TNBC, as well as the main outcomes of this specific immunotherapy.

\section{Atezolizumab in the Treatment of Metastatic TNBC}

The first relevant clinical trial of immunotherapy in TNBC was Impassion 130. This trial included 451 patients (median followup, 12.9 months). In the intention-to-treat analysis, the median Progression-Free Survival (PFS) was 7.2 months with atezolizumab plus nab-paclitaxel, as compared with 5.5 months with placebo plus nab-paclitaxel (hazard ratio for progression or death, 0.80; $95 \%$ Confidence Interval [CI], 0.69 to $0.92 ; \mathrm{P}=0.002$ ); among patients with PD-L1-positive tumors, the median PFS was 7.5 months and 5.0 months, respectively (hazard ratio, $0.62 ; 95 \% \mathrm{CI}, 0.49$ to 0.78 ; $\mathrm{P}<0.001)$. No difference was observed in overall survival (OS) in the intention-to-treat analysis [21.3 months with atezolizumab plus nabpaclitaxel and 17.6 months with placebo plus nab-paclitaxel (hazard ratio for death, $0.84 ; 95 \% \mathrm{CI}, 0.69$ to $1.02 ; \mathrm{P}=0.08)$ ]; among patients with PD-L1-positive tumors, the median OS was 25.0 months and 15.5 months, respectively (hazard ratio, 0.62 ; $95 \% \mathrm{CI}, 0.45$ to 0.86 ).

At ESMO Virtual Congress 2020 an update of overall survival analysis was presented for the PDL-1 + population which confirm the benefit of immunotherapy (3-year survival rates with atezolizumabnab-paclitaxel versus placebo were $36 \%$ and $22 \%$ ). These results have been widely debated since the study design did not allow drawing these conclusions in PDL1 positive patients and it was only planned to verify the OS in PDL-1 positive patients if the data were positive in the intention to treat population.

The Impassion 131 trial also contradicts the findings of the IMpassion130 trial which corroborates the need for further investigations in terms of the use of atezolizumab in the treatment of TNBC [6-9]. In this trial patients were randomly assigned to atezolizumab plus paclitaxel at or to placebo and paclitaxel. The primary endpoint was PFS in the PD-L1-positive population. A 
statistically significant result (based on a HR of 0.62 and median progression-free survival increasing from 5 to 8 months) would lead to testing in the intent-to-treat population. Secondary endpoints, including overall survival, would be formally tested only if previous tests were significant. PFS was not significantly improved by atezolizumab plus paclitaxel vs paclitaxel alone in either the PD-L1positive ( 6.0 vs 5.7 months; $\mathrm{HR}=0.82 ; P=.20$ ) or the intent-to-treat population (5.7 vs 5.6 months; $\mathrm{HR}=0.86$; significance not formally tested for hierarchy). The combination also did not improve OS in the PD-L1-positive group (22.1 vs 28.3 months; HR = 1.12) or the intentto-treat population (19.2 vs 22.8 months; $\mathrm{HR}=1.11$ ).

Reasons for the discrepancy between the studies maybe related to the use of steroids in the premedication for paclitaxel in IMpassion 131. We also must wait for the publication of this trial to check subsequent therapy lines in both arms since an overall survival of 28.3 months in patients treated with chemotherapy was never seen in other trial.

\section{Atezolizumab in Neoadjuvant Context}

NeoTRIP [10] randomly assigned 280 women with early or locally advanced TNBC to receive neoadjuvant therapy with either atezolizumab plus carboplatin/nab-paclitaxel or placebo plus the same chemotherapy. All patients underwent surgery and then received four further cycles of anthracycline-based chemotherapy. pCR rates were not significantly different between the two study arms: $43.5 \%$ with atezolizumab vs $40.8 \%$ with chemotherapy alone. A multivariate analysis showed that the only variable associated with $\mathrm{PCR}$ rate was PD-L1-positive status $(P<.0001)$.

According to this trial, the addition of atezolizumab to the neoadjuvant chemotherapy for patients with TNBC did not improve the rate of the Pathologic Complete Response (pCR) but we will have to wait to see if there is a long-term benefit. Nonetheless, the IMpassion031 trial [11] has proved that the addition of atezolizumab to the neoadjuvant chemotherapy significantly improved the rates of pathologic complete response, regardless of PD-L1 status with an acceptable safety profile. This phase III, randomized patients to receive atezolizumab or placebo with nab-paclitaxel followed by atezolizumab or placebo with dose-dense doxorubicin and cyclophosphamide. pCR was seen in $57.6 \%$ (95\% CI: 49.7, 65.2) of patients in the atezolizumab arm and in $41.1 \%(33.6,48.9)$ in the placebo arm $(\Delta 16.5 \% ; 5.9,27.1$; 1-sided $\mathrm{P}=0.0044$ [significance boundary, 0.0184 ], $\mathrm{P}=0.0085$ for the intersection hypothesis of ITT and PD-L1+ populations). In PD-L1+ pts $(\mathrm{n}=152)$, pCR was seen in $68.8 \%(57.3,78.9)$ vs $49.3 \%(37.6,61.1)$ of pts $(\Delta 19.5 \%$; $4.2,34.8$; 1 -sided $\mathrm{P}=0.021$; not significant). Median EFS was not reached in either arm, but follow up is short (20 months)

One of the possible explanations for the difference seen in these two trials may at least in part be related to the chemotherapy backbone. In the IMpassion031 study, patients received anthracyclines in the neoadjuvant phase, whereas this was given after surgery in NeoTRIP

\section{Atezolizumab in Adjuvant Context}

Regarding the role of atezolizumab in an adjuvant chemotherapy context, there is a trial that is currently being developed, the
IMpassion030 [12], which consists in a global, prospective, randomized, open-label, phase 3 trial that aims to investigate the safety, efficacy, and pharmacokinetic profile of the adjuvant atezolizumab plus standard taxane adjuvant chemotherapy in contrast to the chemotherapy alone in an early stage of TNBC. Essentially, in these specific trial 2300 patients with operable stage II or III TNBC will be randomized. The adjuvant treatment will consist of weekly paclitaxel for 12 weeks, followed by dose dense anthracycline and cyclophosphamide for 4 cycles every 2 weeks or the same chemotherapy regimen given with atezolizumab every 2 weeks, up to a total period of 1 year. The primary endpoint refers to the invasive disease-free survival, while the secondary endpoint also includes the node and lymph status, as well as the overall survival, safety, patient functioning and health related quality of life.

\section{Conclusion}

The main goal of the present study is to fully understand the benefits of atezolizumab during the treatment of TNBC, as well as the main outcomes of this specific immunotherapy. After reviewing several studies, it is possible to conclude that better biomarkers are needed in order to select patients that are more likely to benefit from Immune Checkpoint Inhibitors (ICIs) and to develop new combination therapies to overcome ICI resistance. The existing biomarkers at the moment are basically four, more precisely: PD-L1, Mismatch Repair (MMR) deficiency, Tumor Mutational Burden (TMB), and Tumor Infiltrating Lymphocytes (TILs) [13]. The PD-L1 expression on tumor cells is the most used biomarker to predict immunotherapy benefit in most clinical trials, despite presenting several limitations. We still don't know which cut-off is best, if it should be measured in tumor cells and / or immune cells or in the primary tumor or metastatic lesion. The MMR deficiency rarely occurs in breast cancer, being more common in early-stage diseases. TMB is a measurement of the number of nonsynonymous mutations carried by tumor cells [14]. Still, a high TMB alone does not seem to represent the optimal predictor for immunotherapeutic response in breast cancer, since definition of high TMB lacks standardization, with different thresholds adopted across studies. Lastly, TILs are a well-known prognostic factor in early and advanced stages TNBC, and their assessment is being implemented as a stratification factor in breast cancer immunotherapy trials $[13,15]$. In sum, further investigations are needed, especially with the goal of presenting better biomarkers in order to select patients with TNBC that are more likely to benefit from immunotherapy, including the usage of atezolizumab.

\section{References}

1. Garrido-Castro AC, Lin NU, Polyak K (2019) Insights into molecular classifications of triple-negative breast cancer: Improving patient selection for treatment. Cancer Discov 9: 176-198. [crossref]

2. Denkert C, Minckwitz G, Darb-Esfahani S, Lederer B, Heppner B, et al. (2018) Tumour-infiltrating lymphocytes and prognosis in different subtypes of breast cancer: A pooled analysis of 3771 patients treated with neoadjuvant therapy. Lancet Oncol 19: 40-50. [crossref]

3. Mittendorf E, Philips A, Meric-Bernstam F, Qiao N, Wu Y, et al. (2014) PD-L1 expression in triple-negative breast cancer. Cancer Immunol Res 2: 361-370. [crossref]

4. Luen S, Virassamy B, Savas P, Salgado R., Loi S (2016) The genomic landscape of breast cancer and its interaction with host immunity. Breast 29: 241-250. [crossref] 
5. Schmid P, Adams S, Rugo H, Schneeweiss A, Barrios C, et al. (2018) Atezolizumab and nab-paclitaxel in advanced triple-negative breast cancer. N Engl J Med 379: 21082121. [crossref]

6. Liu S, Camidge D, Gettinger S, Giaccone G, Heist R., et al. (2017) Atezolizumba (atezo) plus platinum-based chemotherapy (chemo) in Non-Small Cell Lung Cancer (NSCLC): Update from a phase Ib study. J Clin Oncol 35: 9092.

7. Jotte R, Cappuzzo F, Vynnychenko I, Stroyakovskiy D, Abreu D, et al. (2018) Impower131: Primary PFS and safety analysis of a randomized phase III study of atezolizumab + carboplatin + paclitaxel or nab-paclitaxel vs carboplatin + nabpaclitaxel as 1L therapy in advanced squamous NSCLC. J Clin Oncol 36: LBA9000.

8. Adams S, Diamond J, Hamilton E, Pohlmann P, Tolaney S, et al. (2019) Atezolizumab plus nab-paclitaxel in the treatment of metastatic triple-negative breast cancer with 2-year survival follow-up: A phase $1 \mathrm{~b}$ clinical trial. JAMA Oncol 5: 334-342. [crossref]

9. Miles D, Gligorov J, André F, Cameron D, Schneeweiss A, et al. (2020) LBA15 primary results from Impassion131, a double-blind placebo-controlled randomized phase III trial of first-line paclitaxel (PAC) \pm atezolizumab (atezo) for unresectable locally advanced/metastatic triple-negative breast cancer (mTNBC). Annals Oncol 31: S1147-S1148.
10. Gianni L, Huang C-S, Egle D, Bermejo B, Zamagni C, et al. (2020) Pathologic complete response (pCR) to neoadjuvant treatment with or without atezolizumab in triple negative, early high-risk and locally advanced breast cancer. NeoTRIPaPDL1 Michelangelo randomized study. Cancer Res 80: Abstract nr GS3-04.

11. Harbeck N, Xhang H, Barrios C, Saji S, Jung K, et al. (2020) IMpassion031: Results from a phase III study of neoadjuvant (neoadj) atezolizumab + chemotherapy in early triple-negative breast cancer (TNBC). Annals Oncol 31: S1142-S1215.

12. Ignatiadis M, McArthur H, Bailey A, Martinez J, Azambuja E, et al. (2019) ALEXANDRA/ IMpassion030: A phase III study of standard adjuvant chemotherapy with or without atezolizumab in early-stage triple-negative breast cancer. Annals of Oncol 30: v97.

13. Keenan T, Tolaney S (2020) Role of immunotherapy in triple-negative breast cancer. J Natl Compr Canc Netw 18: 479-489. [crossref]

14. Barroso-Sousa R., Jain E, Cohen O, Kim D, Buendia-Buendia J, et al. (2020) Prevalence and mutational determinants of high tumor mutation burden in breast cancer. Annals of oncology: official journal of the European Society for Medical Oncology 31: 387-394. [crossref]

15. Marra A, Viale G, Curigliano G (2019) Recent advances in triple negative breast cancer: The immunotherapy era. BMC Medicine 17: 90. [crossref]

\section{Citation:}

Luz P, Bretes L, Arede AJ (2021) The Role of Atezolizumab in the Treatment of Triple-Negative Breast Cancer. ARCH Women Health Care Volume 4(2): 1-3. 